\title{
Medidas do grau de precisão experimental em ensaios de competição de cultivares de milho
}

\author{
Alberto Cargnelutti Filho(1) e Lindolfo Storck ${ }^{(1)}$ \\ (1)Universidade Federal de Santa Maria, Departamento de Fitotecnia, Avenida Roraima, s/no, Bairro Camobi, CEP 97105-900 Santa Maria, \\ RS. E-mail: cargnelutti@pq.cnpq.br, lindolfo@smail.ufsm.br
}

Resumo - O objetivo deste trabalho foi avaliar a adequação de algumas estatísticas como medidas do grau de precisão experimental, e as relações entre elas. Foram usados os dados de produtividade de grãos de 101 ensaios de competição de cultivares de milho (Zea mays L.), realizados no Estado do Rio Grande do Sul, nos anos agrícolas 2002/2003, 2003/2004 e 2004/2005. Foi estudada a relação entre 12 estatísticas, estimadas para cada ensaio, e as análises de correlação de trilha e de agrupamento foram realizadas com base nessas estatísticas. Os limites de classe estabelecidos, a partir das estatísticas acurácia seletiva, herdabilidade, coeficiente de determinação e valor do teste F para cultivar, mostraram-se adequados para estimar o grau de precisão experimental de ensaios de competição de cultivares de milho. Por essas estatísticas, $89 \%$ dos ensaios de competição de cultivares de milho apresentaram precisão alta ou muito alta e $5 \%$ dos ensaios poderiam ser descartados em razão da insuficiência na precisão experimental. As estatísticas acurácia seletiva, herdabilidade, coeficiente de determinação e valor do teste $\mathrm{F}$ para cultivar têm relação direta entre si e são mais adequadas do que o coeficiente de variação e a diferença mínima significativa pelo teste de Tukey, em percentagem da média, para avaliação da precisão experimental em ensaios de competição de cultivares de milho.

Termos para indexação: Zea mays, controle de qualidade, planejamento experimental, produtividade de grãos.

\section{Measures of experimental precision degree in corn cultivar competition trials}

\begin{abstract}
The objective of this work was to evaluate the appropriateness of some statistics as experimental precision measures and the relationships among them. Grain yield data from 101 cultivar competition trials of corn (Zea mays L.), which were carried out in the Rio Grande do Sul state, Brazil, in the agricultural years 2002/2003, 2003/2004 and 2004/2005, were used. Relationship among 12 statistics, estimated from each trial, was studied and correlation, path and cluster analysis were calculated based on these statistics. Class limits established by the statistics selective accuracy, heritability, coefficient of determination and $\mathrm{F}$ test value for cultivar were adequate to estimate the degree of experimental precision in corn yield trials. According to these statistics, $89 \%$ of the experiments showed a high or very high degree of precision and $5 \%$ of experiments could be discarded due to insufficient experimental precision. The statistics selective accuracy, heritability, coefficient of determination and $\mathrm{F}$ test value for cultivar show a direct relationship among them and are more adequate than the coefficient of variation and the significant minimum difference among cultivars by Tukey's test, expressed in percentage of the mean, to evaluate the experimental precision of corn yield trials.
\end{abstract}

Index terms: Zea mays, quality control, experimental planning, grain yield.

\section{Introdução}

O conhecimento dos limites de classe da precisão experimental dos resultados de ensaios de competição de cultivares, e de outros ensaios, é importante para a qualificação das conclusões obtidas. Estatísticas como o coeficiente de variação (Pimentel-Gomes, 1990), índice de variação (Pimentel-Gomes, 1991) ou, como definido posteriormente, coeficiente de precisão
(Storck et al., 2006), e a diferença mínima significativa (DMS) pelo teste de Tukey, em percentagem da média (Lúcio et al., 1999), têm sido utilizadas para essa finalidade. Essas estatísticas apresentam relação inversa com a precisão experimental, ou seja, quanto maior os seus escores menor é a precisão experimental e, consequentemente, maiores diferenças entre as estimativas de médias serão necessárias para que sejam consideradas significativas. 
Faixas de precisão experimental obtidas a partir dessas estatísticas, usando diferentes critérios para se estabelecer os respectivos limites, têm sido propostas para diversas culturas, tipos de experimentos e caracteres (Estefanel et al., 1987; Garcia, 1989; Scapim et al., 1995; Amaral et al., 1997; Lúcio et al., 1997, 1999; Judice et al., 1999, 2002; Lúcio \& Storck, 1999; Clemente \& Muniz, 2002; Costa et al., 2002; Storck et al., 2002; Carvalho et al., 2003; Lima et al., 2004; Mohallem et al., 2008). Entre essas estatísticas, o uso do coeficiente de variação como medida da precisão experimental tem se destacado. No entanto, outras estatísticas, como a acurácia seletiva, a relação entre os coeficientes de variação genético e experimental, o valor do teste $\mathrm{F}$ para o efeito de cultivar, a herdabilidade, o coeficiente de determinação e o índice de diferenciação de Fasoulas (Fasoulas, 1983), têm sido propostas e apresentam propriedades favoráveis para a classificação dessa precisão (Cargnelutti Filho \& Storck, 2007; Resende \& Duarte, 2007). Maiores valores das estatísticas herdabilidade, coeficiente de determinação, valor do teste $\mathrm{F}$ para cultivar e índice de diferenciação de Fasoulas estão associados a maiores variabilidades genéticas e, também, a menores variâncias residuais. Tais estatísticas têm a vantagem de ser independentes da média do ensaio, o que lhes confere adequabilidade como medida da precisão de experimentos (Cargnelutti Filho \& Storck, 2007). Já as estatísticas coeficiente de variação e DMS estão associadas à média e à variância residual, e, por isso, são mais adequadas para a classificação de experimentos com médias semelhantes. Ademais, como estão altamente correlacionadas entre si, fornecem informações redundantes (Cargnelutti Filho \& Storck, 2007).

Faixas de precisão experimental com base em acurácia seletiva e, consequentemente, em valores do teste F para cultivar foram estabelecidas teoricamente por Resende \& Duarte (2007). Essas faixas, porém, não foram avaliadas em um conjunto de ensaios reais. Por outro lado, Cargnelutti Filho \& Storck (2007) avaliaram as estatísticas coeficiente de variação, DMS, herdabilidade, coeficiente de determinação, valor do teste $\mathrm{F}$ para cultivar e índice de diferenciação de Fasoulas como candidatas a medidas da precisão experimental, sem definição das faixas de classificação. A distribuição de frequência nas faixas de precisão da estatística acurácia seletiva e as relações de acurácia seletiva com as estatísticas herdabilidade, coeficiente de determinação, valor do teste $\mathrm{F}$ para cultivar e índice de diferenciação de Fasoulas não são, portanto, ainda conhecidas em um conjunto de ensaios de competição de cultivares. Portanto, é importante o estabelecimento de limites de classe dessas estatísticas, para um melhor controle ou monitoramento da qualidade desse tipo de experimento. Além disso, a precisão experimental dos ensaios de competição de cultivares de milho por essas estatísticas ainda é desconhecida.

O objetivo deste trabalho foi avaliar a adequação de algumas estatísticas como medidas do grau de precisão experimental em ensaios de competição de cultivares de milho, e as relações entre elas, considerando-se a variável produtividade de grãos.

\section{Material e Métodos}

Foram usados os dados de produtividade de grãos, corrigidos para 13\% de umidade, de 101 ensaios de competição de cultivares de milho, realizados no Estado do Rio Grande do Sul, nos anos agrícolas 2002/2003, 2003/2004 e 2004/2005. Os dados foram obtidos em Relatórios e Atas das Reuniões Técnicas Anuais do Milho. Em todos os ensaios, as cultivares foram avaliadas no delineamento blocos ao acaso, com três repetições, e o número de cultivares oscilou entre 9 e 40 . As unidades experimentais foram constituídas de duas fileiras com $5 \mathrm{~m}$ de comprimento, espaçadas em $0,8 \mathrm{~m}$.

Inicialmente, foram feitas análises de variância, para cada um dos 101 ensaios, e anotaram-se as estatísticas: quadrado médio de bloco (QMB), quadrado médio de cultivar $(\mathrm{QMC})$, quadrado médio do erro $(\mathrm{QME})$, valor do teste $\mathrm{F}$ para cultivar $\left(\mathrm{Fc}=\mathrm{QM}_{\mathrm{C}} / \mathrm{QM}_{\mathrm{E}}\right)$, média

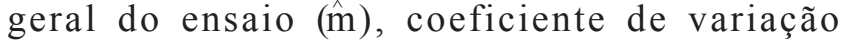
$\left(\mathrm{CV}=100\left(\mathrm{QM}_{\mathrm{E}}\right)^{0,5} / \hat{\mathrm{m}}\right)$ e herdabilidade média $\left(h^{2}=\frac{\left(Q_{C}-Q_{E}\right) / J}{\frac{\left(Q_{C}-Q_{E}\right)}{J}+\frac{Q_{E}}{J}}\right)$

(Cruz, 2001), em que J é o número de repetições.

Em seguida, estimou-se, para cada ensaio, a diferença mínima significativa (DMS) entre as médias de cultivares, pelo teste de Tukey a $5 \%$ de probabilidade, expressa em percentagem da média, por meio da expressão: DMS $=100 \Delta \hat{\mathrm{m}}$, em que $\Delta=\mathrm{q}_{\alpha\left(\mathrm{n} ; \mathrm{GL}_{\mathrm{E}}\right)}\left(\mathrm{QM}_{\mathrm{E}} / \mathrm{J}\right)^{0,5} \mathrm{e} \mathrm{q}_{\alpha(\mathrm{n} ; \mathrm{GL}}$ é o valor crítico para o uso do teste de Tukey; n é o número de cultivares; e $\mathrm{GL}_{\mathrm{E}}$ é o número de graus de liberdade do erro. Determinaram-se, ainda, os valores das estatísticas coeficiente de determinação, calculada pela expressão $\mathrm{R}^{2}=\mathrm{QM}_{\mathrm{C}} /\left(\mathrm{QM}_{\mathrm{C}}+\mathrm{QM}_{\mathrm{E}}\right)$, e a amplitude das médias, estimada pela diferença entre as médias das cultivares 
com maior e menor produtividades $(\mathrm{H}=$ média máxima - média mínima).

O índice de diferenciação de Fasoulas (Fasoulas, 1983) foi estimado pela expressão IF $=200 \sum_{i=1}^{n} \mathrm{~m}_{\mathrm{i}} /$ $[\mathrm{n}(\mathrm{n}-1)]$, em que $\mathrm{n}$ é o número total de cultivares e $\mathrm{m}_{\mathrm{i}} \mathrm{o}$ número de médias que a i-ésima cultivar supera estatisticamente após a aplicação do teste de Tukey. Esse índice fornece o percentual de diferenças significativas entre as médias das cultivares que o teste de comparação múltipla consegue detectar. Em seguida, estimou-se a acurácia seletiva (Resende \& Duarte, 2007), por meio das expressões: $\mathrm{AS}=(1-1 / \mathrm{Fc})^{0,5}$, para $\mathrm{Fc} \geq 1 ; \mathrm{e} A \mathrm{~S}=0$, para $\mathrm{Fc}<1$, em que $\mathrm{Fc}$ é o valor do teste $\mathrm{F}$ para cultivar.

Dessa forma, foram obtidas 12 estatísticas para cada ensaio: $\mathrm{QM}_{\mathrm{B}}, \mathrm{QM}_{\mathrm{C}}, \mathrm{QM}_{\mathrm{E}}$, valor do teste $\mathrm{F}$ para cultivar, média geral do ensaio, coeficiente de variação, herdabilidade, DMS, coeficiente de determinação, amplitude das médias, índice de diferenciação de Fasoulas e acurácia seletiva. A fim de avaliar a eficiência da estatística acurácia seletiva, como medida do grau da precisão experimental dos ensaios, fez-se a análise de correlação linear de Pearson entre acurácia seletiva e as estatísticas $\mathrm{QM}_{\mathrm{B}}, \mathrm{QM}_{\mathrm{C}}, \mathrm{QM}_{\mathrm{E}}$, média geral do ensaio e amplitude das médias, e determinou-se a sua significância por meio do teste t de Student, a 5\% de probabilidade. A seguir, realizaram-se o diagnóstico de multicolinearidade (Cruz, 2001; Cruz \& Carneiro, 2003) e a análise de trilha ("path analysis") tomando-se acurácia seletiva em função das variáveis explicativas $\left(\mathrm{QM}_{\mathrm{B}}, \mathrm{QM}_{\mathrm{C}}, \mathrm{QM}_{\mathrm{E}}\right.$, média geral do ensaio e amplitude das médias).

Determinou-se a matriz de distância euclidiana padronizada entre as estatísticas $\mathrm{QM}_{\mathrm{B}}, \mathrm{QM}_{\mathrm{C}}, \mathrm{QM}_{\mathrm{E}}$, valor do teste $\mathrm{F}$ para cultivar, média geral do ensaio, coeficiente de variação, herdabilidade, DMS, coeficiente de determinação, amplitude das médias, índice de diferenciação de Fasoulas e acurácia seletiva. Essa matriz, em escala relativa, foi utilizada como medida de dissimilaridade para a análise de agrupamento das estatísticas, pelo método hierárquico de Ward (Cruz \& Regazzi, 1997; Cruz, 2001; Cruz \& Carneiro, 2003). Fez-se, a seguir, o diagrama de dispersão das estatísticas coeficiente de variação, DMS, herdabilidade, coeficiente de determinação, valor do teste $\mathrm{F}$ para cultivar, índice de diferenciação de Fasoulas e acurácia seletiva, procurando-se demonstrar as relações entre elas. Calcularam-se os limites das classes de precisão experimental, com base em limites estabelecidos em Resende \& Duarte (2007), e, também, a frequência relativa de ensaios em cada classe.

A fim de comparar as médias das estatísticas $\mathrm{QM}_{\mathrm{B}}$, $\mathrm{QM}_{\mathrm{C}}, \mathrm{QM}_{\mathrm{E}}$, média geral do ensaio e amplitude das médias, para cada classe de precisão experimental, fez-se uma análise de variância, com teste F a 5\% de probabilidade, e comparação das médias por meio do teste (LSD) a, aproximadamente, $5 \%$ de probabilidade. As análises e as estimativas foram realizadas com o auxílio do programa GENES (Cruz, 2001).

\section{Resultados e Discussão}

O diagnóstico de multicolinearidade na matriz de correlações, entre as variáveis explicativas quadrado médio de bloco $\left(\mathrm{QM}_{\mathrm{B}}\right)$, quadrado médio de cultivar $\left(\mathrm{QM}_{\mathrm{C}}\right)$, quadrado médio do erro $\left(\mathrm{QM}_{\mathrm{E}}\right)$, média geral do ensaio e amplitude das médias revelou número de condição igual a 18, o que classifica a matriz como de colinearidade baixa. Assim, a análise de trilha da estatística acurácia seletiva em função das variáveis explicativas pode ser realizada adequadamente (Cruz, 2001; Cruz \& Carneiro, 2003).

A acurácia seletiva apresentou correlação linear $(\mathrm{r}=-0,6430, \mathrm{p} \leq 0,05)$ e efeito direto $(-0,7229)$ com mesmo sinal e de magnitude semelhante ao $\mathrm{QM}_{\mathrm{E}}$ (Tabela 1). Em menor magnitude, a acurácia seletiva apresentou associação linear $(r=0,3256$, $\mathrm{p} \leq 0,05)$ e efeito direto $(0,2801)$ com $\mathrm{QM}_{\mathrm{C}}$, o que revela associação positiva entre essa estatística e variância genotípica. Esses resultados evidenciam que os ensaios com maior acurácia seletiva, em geral, são aqueles com menor variância residual e maior variância genotípica. Constatou-se, ainda, associação positiva entre acurácia seletiva e amplitude das médias $(\mathrm{r}=0,2865, \mathrm{p} \leq 0,05)$, com efeito direto positivo $(0,2688)$, e ausência de associação entre acurácia seletiva e média geral $(\mathrm{r}=-0,1300, \mathrm{p}>0,05)$, com efeito direto muito baixo $(-0,0816)$. Cargnelutti Filho \& Storck (2007) observaram resultados semelhantes, em relação às estatísticas herdabilidade, coeficiente de determinação, valor do teste F para cultivar e índice de diferenciação de Fasoulas. Esses autores destacaram as vantagens das estatísticas herdabilidade, coeficiente de determinação, valor do teste $\mathrm{F}$ para cultivar e índice de diferenciação de Fasoulas, em relação ao coeficiente de variação e à DMS entre as médias das cultivares 
Tabela 1. Estimativas dos coeficientes de correlação de Pearson e respectivas estimativas dos efeitos diretos e indiretos das estatísticas quadrado médio de bloco $\left(\mathrm{QM}_{\mathrm{B}}\right)$, quadrado médio de cultivar $\left(\mathrm{QM}_{\mathrm{C}}\right)$, quadrado médio do erro $\left(\mathrm{QM}_{\mathrm{E}}\right)$, média geral do ensaio ( $\mathrm{m}$ ) e amplitude das médias (H) sobre a estatística acurácia seletiva (AS), para dados de produtividade de grãos de 101 ensaios de competição de cultivares de milho.

\begin{tabular}{|c|c|c|c|c|c|}
\hline Efeito & $\mathrm{QM}_{\mathrm{B}}$ & $\mathrm{QM}_{\mathrm{C}}$ & $\mathrm{QM}_{\mathrm{E}}$ & $\hat{\mathrm{m}}$ & $\mathrm{H}$ \\
\hline Direto sobre AS & $-0,0664$ & 0,2801 & $-0,7229$ & $-0,0816$ & 0,2688 \\
\hline Indireto via $\mathrm{QM}_{\mathrm{B}}$ & & 0,0029 & $-0,0151$ & $-0,0152$ & 0,0034 \\
\hline Indireto via $\mathrm{QM}_{\mathrm{C}}$ & $-0,0121$ & & 0,0576 & 0,1376 & 0,2410 \\
\hline Indireto via $\mathrm{QM}_{\mathrm{E}}$ & $-0,1640$ & $-0,1486$ & & $-0,2932$ & $-0,1895$ \\
\hline Indireto via $\hat{\mathrm{m}}$ & $-0,0186$ & $-0,0401$ & $-0,0331$ & & $-0,0371$ \\
\hline Indireto via $\mathrm{H}$ & $-0,0137$ & 0,2313 & 0,0705 & 0,1223 & \\
\hline Total (correlação de Pearson) & $-0,2748^{*}$ & $0,3256^{*}$ & $-0,6430^{*}$ & $-0,1300^{\mathrm{ns}}$ & $0,2865^{*}$ \\
\hline Coeficiente de determinação & - & - & 0,6619 & - & - \\
\hline
\end{tabular}

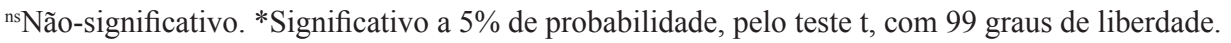

pelo teste de Tukey, expressa em percentagem da média. Logo, ensaios classificados como mais precisos por herdabilidade, coeficiente de determinação, valor do teste $\mathrm{F}$ para cultivar e índice de diferenciação de Fasoulas (maiores escores) não dependeriam da média e estariam relacionadas a menores variâncias residuais $\left(\mathrm{QM}_{\mathrm{E}}\right)$ e maiores variâncias entre tratamentos $\left(\mathrm{QM}_{\mathrm{C}}\right)$. Do ponto de vista da precisão experimental, isso é desejável pois, segundo tais critérios, experimentos com alta variabilidade residual e baixa variabilidade genotípica (pequena variação genética entre as cultivares) tendem a ser eliminados.

Em um dendrograma, grande mudança de nível indica a união de estatísticas heterogêneas (Barroso \& Artes, 2003). Assim, ao se utilizar 50\% de similaridade como critério para definição dos grupos, pelo método de Ward, houve formação de três grupos de estatísticas (Figura 1). As estatísticas acurácia seletiva, coeficiente de determinação, herdabilidade, índice de diferenciação de Fasoulas e valor do teste F para cultivar, destacadas como adequadas para a classificação da precisão experimental, formaram um grupo, o que comprova serem estatísticas similares. Já a DMS e o coeficiente de variação formaram outro grupo. Essas estatísticas, por estarem associadas à média e à variância residual, são medidas adequadas de precisão experimental para ensaios com médias semelhantes (Cargnelutti Filho \& Storck, 2007). Em outro grupo, reuniram-se as estatísticas amplitude das médias, $\mathrm{QM}_{\mathrm{C}}$, média, $\mathrm{QM}_{\mathrm{E}}$ e $\mathrm{QM}_{\mathrm{B}}$, utilizadas como variáveis explicativas na análise de trilha (Cargnelutti Filho \& Storck, 2007). Portanto, pode-se inferir que as estatísticas acurácia seletiva, herdabilidade, coeficiente de determinação, valor do teste $\mathrm{F}$ para cultivar e índice de diferenciação de Fasoulas fornecem informações similares acerca do

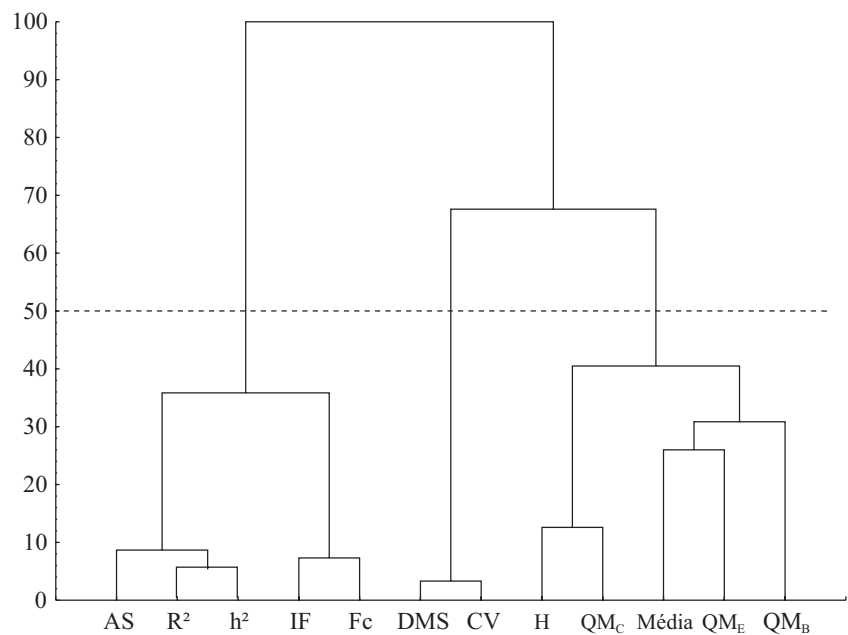

Figura 1. Dendrograma das estatísticas quadrado médio de bloco $\left(\mathrm{QM}_{\mathrm{B}}\right)$, quadrado médio de cultivar $\left(\mathrm{QM}_{\mathrm{C}}\right)$, quadrado médio do erro $\left(\mathrm{QM}_{\mathrm{E}}\right)$, valor do teste $\mathrm{F}$ para cultivar $(\mathrm{Fc})$, média geral do ensaio (média), coeficiente de variação (CV), herdabilidade $\left(\mathrm{h}^{2}\right)$, diferença mínima significativa (DMS) pelo teste de Tukey, em percentagem da média, coeficiente de determinação $\left(\mathrm{R}^{2}\right)$, amplitude das médias $(\mathrm{H})$, índice de diferenciação de Fasoulas (IF) e acurácia seletiva (AS). O dendrograma foi obtido pelo método de Ward, a partir da matriz de distância euclidiana padronizada.

grau de precisão experimental e podem ser consideradas adequadas para tal finalidade, conforme já sustentavam Cargnelutti Filho \& Storck (2007) e Resende \& Duarte (2007).

As distribuições de frequência revelam a possibilidade de as estatísticas coeficiente de variação e DMS ajustaram-se à distribuição normal (Figura 2). A comprovação dessa visualização foi obtida por meio do teste de normalidade de Lilliefors (Sprent \& Smeeton, 2007), que revelou, a 5\% de probabilidade, que elas foram as únicas estatísticas que se ajustaram 
à distribuição normal de probabilidade. As estatísticas herdabilidade, coeficiente de determinação e acurácia seletiva apresentaram assimetria à esquerda (negativa) e as estatísticas valor do teste F para cultivar e índice de diferenciação de Fasoulas, assimetria à direita (positiva), o que comprova a rejeição da hipótese de normalidade do teste de Lilliefors. Diante disso, há necessidade de se estudar critérios para a definição dos limites de classe da precisão de experimentos, para estatísticas que não seguem a distribuição normal, o que foi destacado por Cargnelutti Filho \& Storck (2007).

Há relações diretas e não lineares entre as estatísticas acurácia seletiva, valor do teste $\mathrm{F}$ para cultivar, herdabilidade e coeficiente de determinação (Figura 2), que ainda podem ser exploradas. A acurácia seletiva, estimada por meio da expressão AS $=[1-(1 / \mathrm{Fc})]^{0,5}$, conforme demonstram Resende \& Duarte (2007), apresenta relação direta com o valor do teste $\mathrm{F}$ para cultivar, ou seja: $\mathrm{Fc}=1 /\left(1-\mathrm{AS}^{2}\right)$. Já a herdabilidade, usada em médias de cultivares, em um delineamento de blocos ao acaso com $\mathrm{J}$ repetições, é estimada pela expressão:

$\mathrm{h}^{2}=\frac{\left(\mathrm{QM}_{\mathrm{C}}-\mathrm{QM}_{\mathrm{E}}\right) / \mathrm{J}}{\frac{\left(\mathrm{QM}_{\mathrm{C}}-\mathrm{QM}_{\mathrm{E}}\right)}{\mathrm{J}}+\frac{\mathrm{QM}_{\mathrm{E}}}{\mathrm{J}}}=\frac{\mathrm{QM}_{\mathrm{C}}-\mathrm{QM}_{\mathrm{E}}}{\mathrm{QM}_{\mathrm{C}}}$;

ou, ainda, por: $\mathrm{h}^{2}=1-\left(\mathrm{QM}_{\mathrm{E}} / \mathrm{QM}_{\mathrm{C}}\right)$. Usando-se $\mathrm{Fc}$ $=\mathrm{QM}_{\mathrm{C}} / \mathrm{QM}_{\mathrm{E}}$ e $\mathrm{h}^{2}=1-(1 / \mathrm{Fc})$, em substituição na expressão de acurácia seletiva, tem-se como resultado:

$\mathrm{AS}=\left(\mathrm{h}^{2}\right)^{0,5}$ ou $\mathrm{h}^{2}=\mathrm{AS}^{2}$.

Ainda, o coeficiente de determinação, estimado por $\mathrm{R}^{2}=\left(\mathrm{QM}_{\mathrm{C}}\right) /\left(\mathrm{QMC}+\mathrm{QM}_{\mathrm{E}}\right)$, pode ser dividido por
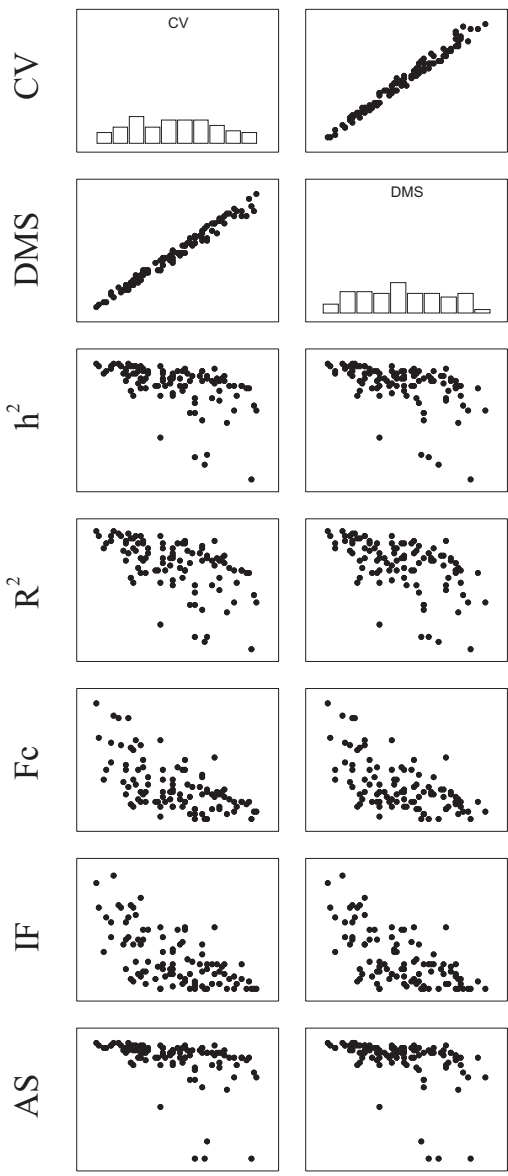

$\mathrm{CV}$
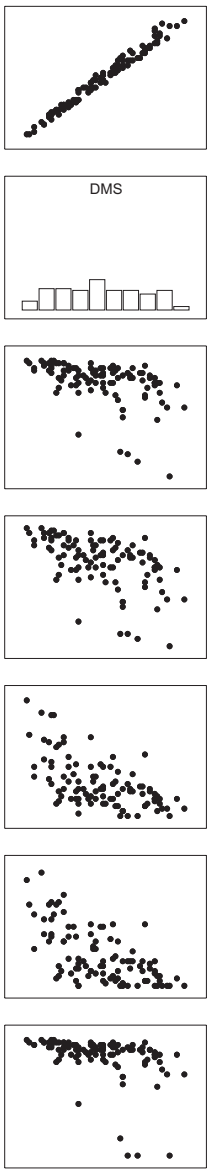

DMS


$\mathrm{h}^{2}$

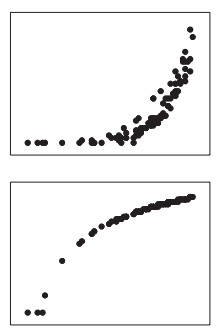

$\mathrm{R}^{2}$
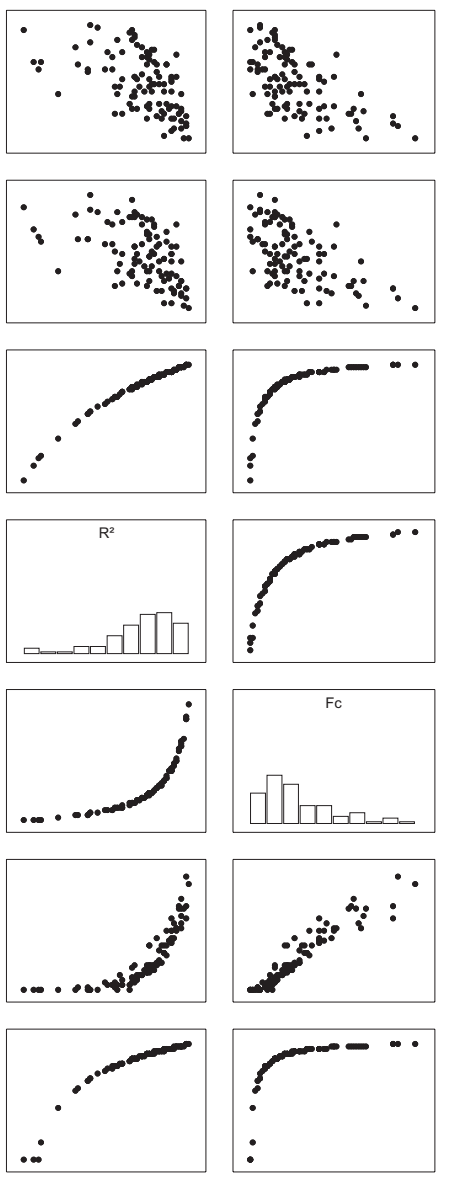

Fc
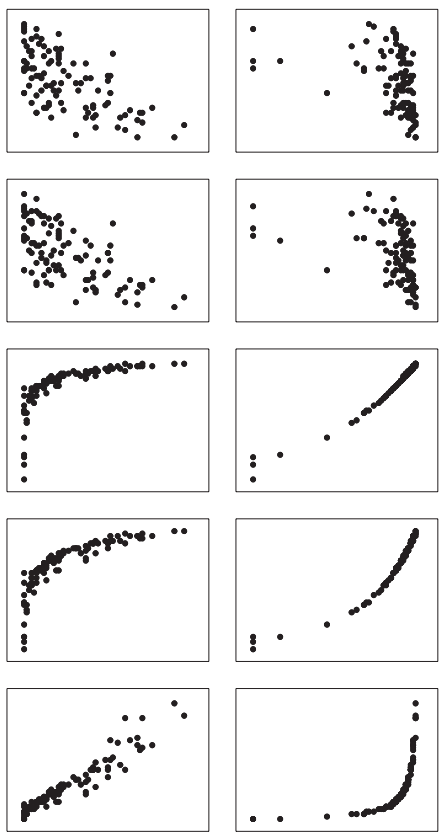

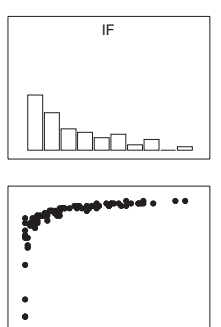

IF

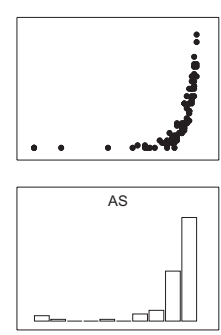

AS

Figura 2. Matriz com a distribuição de frequência (na diagonal) e gráficos de dispersão entre as estatísticas coeficiente de variação (CV), diferença mínima significativa (DMS) pelo teste de Tukey, em percentagem da média, herdabilidade $\left(\mathrm{h}^{2}\right)$, coeficiente de determinação $\left(\mathrm{R}^{2}\right)$, valor do teste $\mathrm{F}$ para cultivar $(\mathrm{Fc})$, índice de diferenciação de Fasoulas (IF) e acurácia seletiva (AS) (fora da diagonal), obtidos de 101 ensaios de competição de cultivares de milho, em relação à produtividade de grãos. 
$\mathrm{QM}_{\mathrm{C}}$, para obter $\mathrm{R}^{2}=\frac{1}{\left(1+\frac{\mathrm{QM}_{\mathrm{E}}}{\mathrm{QM}_{\mathrm{C}}}\right)}=\frac{1}{\left(1+\frac{1}{\mathrm{Fc}}\right)}$.

Isolando-se $(1 / \mathrm{Fc})$, obtém-se $\left[(1 / \mathrm{Fc})=\left(1 / \mathrm{R}^{2}\right)-1\right]$. Substituindo $(1 / \mathrm{Fc})$ na expressão de acurácia seletiva, têm-se:

$\mathrm{AS}=\sqrt{1-\left(\frac{1}{\mathrm{R}^{2}}-1\right)}=\sqrt{2-\frac{1}{\mathrm{R}^{2}}}$, ou seja, $\mathrm{R}^{2}=\frac{1}{2-\mathrm{AS}^{2}}$.

Assim, demonstra-se também que as estatísticas herdabilidade, coeficiente de determinação e valor do teste F para cultivar podem ser estimadas diretamente, a partir do conhecimento da acurácia seletiva. Isso é particularmente importante para se definir os limites das classes de precisão experimental com base em valor do teste F para cultivar (Cargnelutti Filho \& Storck, 2007; Resende \& Duarte, 2007), herdabilidade e coeficiente de determinação (Cargnelutti Filho \& Storck, 2007), a partir dos limites de classe de acurácia seletiva, estabelecidos em Resende \& Duarte (2007) (Tabela 2). Não se estabeleceu limites de faixas de classificação com base no índice de diferenciação de Fasoulas, por não apresentar relação direta com acurácia seletiva e, ainda, por ser uma estatística de qualidade um pouco inferior às demais, como medida do grau de precisão experimental (Cargnelutti Filho \& Storck, 2007).
Pelas estatísticas analisadas, $89 \%$ dos ensaios apresentaram precisão alta ou muito alta $(\mathrm{AS} \geq 0,70$; $h^{2} \geq 0,49 ; R^{2} \geq 0,6623$ e $F c \geq 1,9608$ ) e $5 \%$ dos ensaios poderiam ser descartados por apresentarem precisão experimental insuficiente (Tabela 2). Com base nas estatísticas coeficiente de variação e DMS, Lúcio et al. (1999) e Cargnelutti Filho \& Storck (2007) encontraram maiores frequências de experimentos nas classes de baixa e muito baixa precisão experimental. Essa divergência de resultados pode ser explicada pelas diferentes estatísticas utilizadas para a classificação da precisão experimental (Cargnelutti Filho \& Storck, 2007). Observou-se ainda que, pelas diferentes estatísticas investigadas (Tabela 3), a tendência é de se classificar como mais precisos os experimentos que têm maior variabilidade genética $\left(\mathrm{QM}_{\mathrm{C}}\right)$ e menor variação residual $\left(\mathrm{QM}_{\mathrm{E}}\right)$, isto é, aqueles que discriminam mais as cultivares, independentemente da média geral do experimento (Cargnelutti Filho \& Storck, 2007; Resende \& Duarte, 2007). Dado que, entre as estatísticas avaliadas, somente acurácia seletiva, herdabilidade, coeficiente de determinação e valor do teste $\mathrm{F}$ para cultivar consideram simultaneamente essas informações, pode-se inferir que essas estatísticas são mais adequadas para se avaliar a precisão nos ensaios de competição de cultivares de milho.

Tabela 2. Limites das classes de precisão experimental, em relação à produtividade de grãos, para as estatísticas acurácia seletiva (AS), herdabilidade $\left(\mathrm{h}^{2}\right)$, coeficiente de determinação $\left(\mathrm{R}^{2}\right)$ e valor do teste $\mathrm{F}$ para cultivar $(\mathrm{Fc})$ e a frequência relativa $\left(\mathrm{fr}_{\mathrm{i}}\right)$ de ensaios de competição de cultivares de milho em cada classe.

\begin{tabular}{|c|c|c|c|c|c|c|}
\hline \multirow{3}{*}{$\begin{array}{l}\text { Precisão } \\
\text { experimental }\end{array}$} & \multirow{3}{*}{$\begin{array}{c}\text { AS }=\sqrt{1-\frac{1}{F c}} \\
\text { (Resende \& } \\
\text { Duarte, 2007) }\end{array}$} & \multirow{3}{*}{$\begin{array}{c}\mathrm{h}^{2}=\mathrm{AS}^{2} \\
(\text { Cargnelutti Filho } \\
\text { \& Storck, 2007) }\end{array}$} & \multirow{2}{*}{$\mathrm{R}^{2}=$} & 1 & 1 & \multirow[t]{3}{*}{$\mathrm{fr}_{\mathrm{i}}(\%)$} \\
\hline & & & & $2-\mathrm{AS}^{2}$ & $1-\mathrm{AS}^{2}$ & \\
\hline & & & \multicolumn{2}{|c|}{$\begin{array}{l}\text { (Cargnelutti Filho \& } \\
\text { Storck, 2007) }\end{array}$} & $\begin{array}{l}\text { (Cargnelutti Filho \& Storck, } \\
\text { 2007; Resende \& Duarte, } \\
\text { 2007) }\end{array}$ & \\
\hline Muito alta & $\geq 0,90$ & $\geq 0,81$ & \multicolumn{2}{|c|}{$\geq 0,8403$} & $\begin{array}{c}\geq 5,2632 \\
\geq 19608\end{array}$ & 42,57 \\
\hline Alta & $\geq 0,70$ e $<0,90$ & $\geq 0,49$ e $<0,81$ & \multicolumn{2}{|c|}{$\geq 0,6623 \mathrm{e}<0,8403$} & $\geq 1,9608$ e $<5,2632$ & 46,53 \\
\hline Moderada & $\geq 0,50$ e $<0,70$ & $\geq 0,25$ e $<0,49$ & \multicolumn{2}{|c|}{$\geq 0,5714$ e $<0,6623$} & $\geq 1,3333$ e $<1,9608$ & 5,94 \\
\hline Baixa & $<0,5$ & $<0,25$ & \multicolumn{2}{|c|}{$<0,5714$} & $<1,3333$ & 4,95 \\
\hline
\end{tabular}

Tabela 3. Média dos quadrados médios de bloco $\left(\mathrm{QM}_{\mathrm{B}}\right)$, de cultivar $\left(\mathrm{QM}_{\mathrm{C}}\right)$ e do erro $\left(\mathrm{QM}_{\mathrm{E}}\right)$, da média geral do ensaio $\left(\hat{\mathrm{m}}, \mathrm{kg} \mathrm{ha}^{-1}\right)$ e da amplitude das médias de cultivares $\left(\mathrm{H}, \mathrm{kg} \mathrm{ha}^{-1}\right)$ em cada classe da precisão experimental de 101 ensaios de competição de cultivares de milho, em relação à produtividade de grãos ${ }^{(1)}$.

\begin{tabular}{lccccc}
\hline Precisão experimental & $\mathrm{QM}_{\mathrm{B}}$ & $\mathrm{QM}_{\mathrm{C}}$ & $\mathrm{QM}_{\mathrm{E}}$ & $\hat{\mathrm{m}}$ \\
\hline Muito alta & $2.174 .203 \mathrm{~b}$ & $4.461 .149 \mathrm{a}$ & $496.571 \mathrm{c}$ & $7.051^{\mathrm{ns}}$ & $4.531 \mathrm{a}$ \\
Alta & $4.534 .497 \mathrm{~b}$ & $3.251 .302 \mathrm{ab}$ & $891.465 \mathrm{~b}$ & 6.620 & $3.843 \mathrm{a}$ \\
Moderada & $6.682 .152 \mathrm{~b}$ & $3.221 .227 \mathrm{ab}$ & $1.904 .236 \mathrm{a}$ & 7.869 & $4.303 \mathrm{a}$ \\
Baixa & $14.196 .637 \mathrm{a}$ & $1.764 .266 \mathrm{~b}$ & $1.823 .334 \mathrm{a}$ & 8.351 & $2.792 \mathrm{~b}$ \\
\hline
\end{tabular}

${ }^{(1)}$ Médias seguidas por letras distintas, na coluna, diferem entre si pelo teste $t$ (LSD), a $5 \%$ de probabilidade. ${ }^{\text {ns}}$ Não-significativo. 


\section{Conclusões}

1. As estatísticas acurácia seletiva, herdabilidade, coeficiente de determinação e valor do teste F para cultivar têm relação direta entre si e são mais adequadas do que o coeficiente de variação e a diferença mínima significativa (DMS) pelo teste de Tukey, em percentagem da média, para se avaliar a precisão experimental em ensaios de competição de cultivares de milho.

2. Os limites de classe estabelecidos, a partir das estatísticas acurácia seletiva, herdabilidade, coeficiente de determinação e valor do teste $\mathrm{F}$ para cultivar são adequados para estimar o grau de precisão experimental de ensaios de competição de cultivares de milho.

\section{Agradecimentos}

Ao Conselho Nacional de Desenvolvimento Científico e Tecnológico, pela concessão de bolsas; ao Engenheiro Agrônomo José Paulo Guadagnin, da Fundação Estadual de Pesquisa Agropecuária, pela coordenação dos ensaios de avaliação de cultivares de milho no Estado do Rio Grande do Sul; aos pesquisadores de outras instituições, que auxiliaram na realização desses ensaios.

\section{Referências}

AMARAL, A.M.; MUNIZ, J.A.; SOUZA, M. Avaliação do coeficiente de variação como medida da precisão na experimentação com citros. Pesquisa Agropecuária Brasileira, v.32, p.1221-1225, 1997.

BARROSO, L.P.; ARTES, R. Análise multivariada. Lavras: Ufla, 2003. 151p.

CARGNELUTTI FILHO, A.; STORCK, L. Estatísticas de avaliação da precisão experimental em ensaios de cultivares de milho. Pesquisa Agropecuária Brasileira, v.42, p.17-24, 2007.

CARVALHO, C.G.P. de; ARIAS, C.A.A.; TOLEDO, J.F.F. de; ALMEIDA, L.A. de; KIIHL, R.A. de S.; OLIVEIRA, M.F. de; HIROMOTO, D.M.; TAKEDA, C. Proposta de classificação dos coeficientes de variação em relação à produtividade e altura da planta de soja. Pesquisa Agropecuária Brasileira, v.38, p.187-193, 2003.

CLEMENTE, A.L.; MUNIZ, J.A. Avaliação do coeficiente de variação em experimentos com gramíneas forrageiras. Ciência e Agrotecnologia, v.26, p.197-203, 2002.

COSTA, N.H. de A.D.; SERAPHIN, J.C.; ZIMMERMANN, F.J.P. Novo método de classificação de coeficientes de variação para a cultura do arroz de terras altas. Pesquisa Agropecuária Brasileira, v.37, p.243-249, 2002.

CRUZ, C.D. Programa GENES: versão Windows: aplicativo computacional em genética e estatística. Viçosa: UFV, 2001. 648p.

CRUZ, C.D.; CARNEIRO, P.C.S. Modelos biométricos aplicados ao melhoramento genético. Viçosa: UFV, 2003. v.2. 585p.
CRUZ, C.D.; REGAZZI, A.J. Modelos biométricos aplicados ao melhoramento genético. 2.ed. Viçosa: UFV, 1997. 390p.

ESTEFANEL, V.; PIGNATARO, I.A.B.; STORCK, L. Avaliação do coeficiente de variação de experimentos com algumas culturas agrícolas. In: SIMPÓSIO DE ESTATÍSTICA APLICADA À EXPERIMENTAÇÃOAGRONÔMICA, 2.; REUNIÃOANUALDA REGIÃO BRASILEIRA DA SOCIEDADE INTERNACIONAL DE BIOMETRIA, 32., 1987, Londrina. Anais. Londrina: Universidade Estadual de Londrina, 1987. p.115-131.

FASOULAS, A.C. Rating cultivars and trials in applied plant breeding. Euphytica, v.32, p.939-943, 1983.

GARCIA, C.H. Tabelas para classificação do coeficiente de variação. Piracicaba: Ipef, 1989. 12p. (Circular técnica, 171).

JUDICE, M.G.; MUNIZ, J.A.; AQUINO, L.H. de; BEARZOTI, E. Avaliação da precisão experimental em ensaios com bovinos de corte. Ciência e Agrotecnologia, v.26, p.1035-1040, 2002.

JUDICE, M.G.; MUNIZ, J.A.; CARVALHEIRO, R. Avaliação do coeficiente de variação na experimentação com suínos. Ciência e Agrotecnologia, v.23, p.170-173, 1999.

LIMA, L.L.; NUNES, G.H.S.; BEZERRA NETO, F. Coeficientes de variação de algumas características do meloeiro: uma proposta de classificação. Horticultura Brasileira, v.22, p.14-17, 2004.

LÚCIO, A.D.; STORCK, L. O manejo das culturas interfere no erro experimental. Pesquisa Agropecuária Gaúcha, v.5, p.311-316, 1999.

LÚCIO, A.D.; STORCK, L.; BANZATTO, D.A. Classificação dos experimentos de competição de cultivares quanto a sua precisão. Pesquisa Agropecuária Gaúcha, v.5, p.99-103, 1999.

LÚCIO, A.D.; STORCK, L.; MARCHEZAN, E. Verifique a qualidade de seu experimento. Lavoura Arrozeira, v.50, p.23-25, 1997.

MOHALLEM, D.F.; TAVARES, M.; SILVA, P.L.; GUIMARÃES, E.C.; FREITAS, R.F. Avaliação do coeficiente de variação como medida da precisão em experimentos com frangos de corte. Arquivo Brasileiro de Medicina Veterinária e Zootecnia, v.60, p.449-453, 2008.

PIMENTEL-GOMES, F. Curso de estatística experimental. 13.ed. Piracicaba: Nobel, 1990. 468p.

PIMENTEL-GOMES, F. O índice de variação, um substituto vantajoso do coeficiente de variação. Piracicaba: Ipef, 1991. 4p. (Circular técnica, 178).

RESENDE, M.D.V. de; DUARTE, J.B. Precisão e controle de qualidade em experimentos de avaliação de cultivares. Pesquisa Agropecuária Tropical, v.37, p.182-194, 2007.

SCAPIM, C.A.; CARVALHO, C.G.P. de; CRUZ, C.D. Uma proposta de classificação dos coeficientes de variação para a cultura do milho. Pesquisa Agropecuária Brasileira, v.30, p.683-686, 1995.

SPRENT, P.; SMEETON, N.C. Applied nonparametric statistical methods. $4^{\text {th }}$ ed. Boca Raton: Chapman \& Hall, 2007. 530p.

STORCK, L.; GARCIA, D.C.; LOPES, S.J.; ESTEFANEL, V. Experimentação vegetal. Santa Maria: UFSM, 2006. 198p.

STORCK, L.; LÚCIO, A.D.; SANTOS, P.M.; CARVALHO, M.P.; CARDINAL, Á.B.B. Precisão experimental em erva-mate (Ilex paraguariensis St. Hil.). Ciência Florestal, v.12, p.159-161, 2002.

Recebido em 23 de outubro de 2008 e aprovado em 30 de janeiro de 2009 